Vasile Ene, Ovidius University Constanţa, Romania Current address: 23 August 8717, Jud. Constanţa, Romania

e-mail:ene@s23aug.sfos.ro or ene@univ-ovidius.ro

\title{
AN ELEMENTARY PROOF OF THE BANACH-ZARECKI THEOREM
}

\begin{abstract}
In this paper we shall give a new, elementary proof of the BanachZarecki theorem, based on the following classical result [3] (p. 183): If $\left\{A_{i}\right\}_{i}$ is a sequence of decreasing sets in a measurable space $(X, \mathcal{M}, \mu)$ and $\mu\left(A_{1}\right)<+\infty$ then $\mu\left(\cap_{i} A_{i}\right)=\lim _{i \rightarrow \infty} \mu\left(A_{i}\right)$.
\end{abstract}

There is a very rich literature concerning the Banach-Zarecki Theorem, such as the books of Saks [5] (p. 227), Natanson [4] (p. 250), Foran [3] (p. 357), Ene [1] (pp. 81, 104) and a paper of Varberg [6] (p. 835). This theorem asserts that if a continuous and $V B$ function satisfies Lusin's condition $(N)$ on an interval then it is also $A C$ on that interval.

The proofs in [5], [3], [6] and [1] (p. 81) are based on the following result (see Theorem 6.5 of [5], p. 227; Theorem 1 of [6], p. 834; Theorem 8.1 of [3]): If a function $F$ is derivable at every point of a measurable set $D$, then $m^{*}(F(D)) \leq(\mathcal{L}) \int_{D}\left|F^{\prime}(x)\right| d x$.

In [4], the Banach-Zarecki Theorem is proved in a totally different way, namely using Lebesgue's Convergence Theorem as well as the fact that the Banach indicatrix for a continuous and $V B$ function on $[a, b]$ is summable (see Theorem 3 of [4], p. 225).

In [1] (p. 104), the Banach-Zarecki Theorem is a consequence of some general notion $\left(A C_{\infty}, V B_{\infty}\right.$ etc.). Here the Banach indicatrix has also an important role, but the proof is different from that in [4].

In this paper we shall give a new, elementary proof of the Banach-Zarecki theorem, based on the following classical result [3] (p. 183): If $\left\{A_{i}\right\}_{i}$ is a sequence of decreasing sets in a measurable space $(X, \mathcal{M}, \mu)$ and $\mu\left(A_{1}\right)<+\infty$ then $\mu\left(\cap_{i} A_{i}\right)=\lim _{i \rightarrow \infty} \mu\left(A_{i}\right)$.

Key Words: $(N), V B, A C$, Banach-Zarecki Theorem

Mathematical Reviews subject classification: 26A45, 26A46, 26A48

Received by the editors February 27, 1997 
Let $m(A)$ denote the Lebesgue measure of the set $A$, whenever $A \subset \mathbb{R}$ is Lebesgue measurable, and $m^{*}(X)$ the outer measure of the set $X$. We denote by $\mathcal{O}(F ;[a, b])$ the oscillation of the function $F$ on $[a, b]$, and by $V(F ;[a, b])$ the variation of $F$ on $[a, b]$. For the definitions of $V B$ and $A C$ see [5].

Lemma 1. Let $f: X \mapsto Y$ and $Y_{1}=\left\{y \in Y: f^{-1}(y)\right.$ contains more than one point $\}$. If $\left(X_{i}\right)_{i \in I}$ is a family of subsets of $X$ then

$$
\left(\cap_{i \in I} f\left(X_{i}\right)\right) \backslash Y_{1} \subseteq f\left(\cap_{i \in I} X_{i}\right) \subseteq \cap_{i \in I} f\left(X_{i}\right) .
$$

Proof. Let $y \in\left(\cap_{i \in I} f\left(X_{i}\right)\right) \backslash Y_{1}$. Then there exists a unique point $x \in X$ such that $y=f(x)$. Since $y \in f\left(X_{i}\right)$ for each $i \in I$, it follows that there exists $x_{i} \in X_{i}$ such that $f\left(x_{i}\right)=y$, so $x_{i}=x$. We obtain that $x \in X_{i}$ for each $i \in I$, hence $x \in \cap_{i \in I} X_{i}$. It follows that $y=f(x) \in f\left(\cap_{i \in I} X_{i}\right)$. The last (well-known) inclusion is easy to verify.

Lemma 2. Let $F:[a, b] \mapsto \mathbb{R}$ be an increasing function and let $B=\{y \in$ $[F(a), F(b)]: F^{-1}(y)$ contains more than one point $\}$. Then:

(i) $B$ is at most countable, hence $B$ is a Borel set;

(ii) If $Z \subset(a, b)$ is a $G_{\delta}$-set then $F(Z)$ is a Borel set.

Moreover, if $Z=\cap_{i=1}^{\infty} G_{i}$, where each $G_{i}$ is an open set, then

$$
m(F(Z))=m\left(\cap_{i=1}^{\infty} F\left(G_{i}\right)\right) .
$$

Proof. (i) For $y \in B$ let $x_{y}^{\prime}=\inf \left(F^{-1}(y)\right)$ and $x_{y}^{\prime \prime}=\sup \left(F^{-1}(y)\right)$. By hypotheses $\emptyset \neq\left(x_{y}^{\prime}, x_{y}^{\prime \prime}\right) \subset F^{-1}(y)$. Since

$$
\left(x_{y_{1}}^{\prime}, x_{y_{1}}^{\prime \prime}\right) \cap\left(x_{y_{2}}^{\prime}, x_{y_{2}}^{\prime \prime}\right)=\emptyset \quad \text { whenever } \quad y_{1} \neq y_{2},
$$

it follows that $B$ is at most countable. have

(ii) Let $Z=\cap_{i=1}^{\infty} G_{i}$, where each $G_{i}$ is an open set. Then by Lemma 1 we

$$
\left(\cap_{i=1}^{\infty} F\left(G_{i}\right)\right) \backslash B \subseteq F\left(\cap_{i=1}^{\infty} G_{i}\right) \subseteq \cap_{i=1}^{\infty} F\left(G_{i}\right) .
$$

Since $F$ is increasing, each $F\left(G_{i}\right)$ is a countable union of intervals (some of them might be degenerate). Hence each $F\left(G_{i}\right)$ is a Borel set and by (1), $F\left(\cap_{i=1}^{\infty} G_{i}\right)$ is also a Borel set.

The last part follows by (i) and (1)

Lemma 3. Let $F:[a, b] \mapsto \mathbb{R}$ be a continuous and increasing function. Then $F \in(N)$ on $[a, b]$ if and only if $m(F(Z))=0$ whenever $Z$ is a compact subset of $[a, b]$ and $m(Z)=0$. 
ProOF. " $\Rightarrow$ " This is obvious.

" $\Leftarrow$ " Suppose on the contrary that $F \notin(N)$ on $[a, b]$. Then there exists a set $Z \subset[a, b]$, with $m(Z)=0$ such that $m^{*}(F(Z))>0$. We may suppose without loss of generality that $Z$ is of $G_{\delta}$-type (because for $Z$ there exists a $G_{\delta}$-set $Z_{1}$ of measure zero, such that $Z \subset Z_{1}$, so $\left.m^{*}\left(F\left(Z_{1}\right)\right)>0\right)$. Then $F(Z)$ is a Borel set of positive measure (see Lemma 2). Let $K$ be a compact subset of $F(Z)$ of positive measure. Then $K_{1}:=F^{-1}(K)$ is a compact subset of $Z$ (because $F$ is continuous), and $F\left(K_{1}\right)=K$, a contradiction.

Remark 1. In [2], Foran introduces the following condition: A function is said to satisfy condition $\left(N^{\prime}\right)$ provided the image of closed sets of measure 0 is of measure 0 .

Using this condition, Lemma 3 from above can be stated as follows: Let $F:[a, b] \mapsto \mathbb{R}$ be a continuous and increasing function. Then $F \in(N)$ if and only if $F \in\left(N^{\prime}\right)$.

Foran showed that conditions $(N)$ and $\left(N^{\prime}\right)$ coincide for Baire functions (in fact his results are much stronger). Hence Lemma 3 is a special case of this result. On the other hand, our proof in this particular case is elementary.

Lemma 4. Let $F:[a, b] \mapsto \mathbb{R}$ be such that $F([a, b])$ is an interval. Suppose that $P$ is a perfect set containing the points $a$ and $b$, and let $\left\{\left(a_{i}, b_{i}\right)\right\}_{i}$ be the intervals contiguous to $P$. Then

$$
|F(b)-F(a)| \leq m(F([a, b])) \leq m^{*}(F(P))+\sum_{i=1}^{\infty} \mathcal{O}\left(F ;\left[a_{i}, b_{i}\right]\right) .
$$

Proof. We have

$$
F([a, b])=F\left(P \cup\left(\cup_{i=1}^{\infty}\left(a_{i}, b_{i}\right)\right)=F(P) \cup\left(\cup_{i=1}^{\infty} F\left(\left(a_{i}, b_{i}\right)\right)\right),\right.
$$

hence

$$
\begin{aligned}
|F(b)-F(a)| \leq & m\left(F([a, b]) \leq m^{*}(F(P))+\sum_{i=1}^{\infty} m^{*}\left(F\left(\left(a_{i}, b_{i}\right)\right)\right) \leq\right. \\
& \leq m^{*}(F(P))+\sum_{i=1}^{\infty} \mathcal{O}\left(F ;\left[a_{i}, b_{i}\right]\right)
\end{aligned}
$$

Lemma 5. ([4], p. 224). Let $f:[a, b] \mapsto \mathbb{R}$ be a continuous function. Subdivide $[a, b]$ by means of the points

$$
x_{o}=a<x_{1}<x_{2}<\cdots<x_{n}=b \text { with } \max \left(x_{k+1}-x_{k}\right)=\lambda,
$$


and form the sums

$$
V=\sum_{k=0}^{n-1}\left|f\left(x_{k+1}\right)-f\left(x_{k}\right)\right| .
$$

If $\lambda \rightarrow 0$ then each of the sums $V$ tends to the total variation $V(f ;[a, b])$ of the function $f(x)$ (we do not suppose that the variation is finite).

Lemma 6. Let $F:[a, b] \mapsto \mathbb{R}$ be a continuous and $V B$ function, and let $H:[a, b] \mapsto \mathbb{R} . H(x)=V(F ;[a, x])$. Then $F \in(N)$ if and only if $H \in(N)$.

Proof. " $\Rightarrow$ " Clearly $H$ is increasing and continuous on $[a, b]$ (see Theorem 1 of [4], p. 223). Suppose on the contrary that $H \notin(N)$. By Lemma 3 it follows that there exists a compact set $Z \subset[a, b]$ of measure zero, such that $H(Z)$ is a compact set (because $H$ is continuous) of positive measure. Let $c=\inf (Z)$, $d=\sup (Z)$. We may suppose without loss of generality that $Z$ is a perfect set (if necessary eliminating the isolated points of $Z$, that are at most countable). Let $\left\{\left(a_{i}, b_{i}\right)\right\}_{i}$ be the intervals contiguous to $Z$. Let $\alpha=m(H(Z))>0$. For each positive integer $n$ let $\left[c_{i}, d_{i}\right], i=1,2, \ldots, n$ be the closed subintervals of $[c, d]$ left after extracting the open intervals $\left(a_{i}, b_{i}\right), i=1,2, \ldots, n-1$. Let $\lambda_{n}=\max _{i=1}^{n}\left(d_{i}-c_{i}\right)$. Since $m(Z)=0$ it follows that $\lim _{n \rightarrow \infty} \lambda_{n}=0$. Let $c=x_{o}<x_{1}<x_{2}<\cdots<x_{p}=d$ be a division of $[c, d]$ that contains all $c_{i}$ and $d_{i}$, each $\left(c_{i}, d_{i}\right)$ containing no $x_{j}$, and such that $x_{j}-x_{j-1}<\lambda_{n}$, for each $j=1,2, \ldots, p$. Let

$$
S_{n}:=\sum_{j=1}^{p}\left|F\left(x_{j}\right)-F\left(x_{j-1}\right)\right|
$$

and

$$
V_{n}:=\sum_{i=1}^{n}\left|F\left(d_{i}\right)-F\left(c_{i}\right)\right|+\sum_{i=1}^{n-1} V\left(F ;\left[a_{i}, b_{i}\right]\right) .
$$

Then $S_{n} \leq V_{n} \leq V:=V(F ;[c, d])$. By Lemma 5 we have

$$
\lim _{n \rightarrow \infty} S_{n}=V, \quad \text { so } \quad \lim _{n \rightarrow \infty} V_{n}=V .
$$

It follows that there exists a positive integer $n_{o}$ such that

$$
V_{n}>V-\frac{\alpha}{2}, \quad \text { whenever } \quad n \geq n_{o} .
$$


By Theorem 5 of [4], p. 217, we obtain

$$
\begin{aligned}
V & =\sum_{i=1}^{n} V\left(F ;\left[c_{i}, d_{i}\right]\right)+\sum_{i=1}^{n-1} V\left(F ;\left[a_{i}, b_{i}\right]\right)= \\
& =\sum_{i=1}^{n}\left(H\left(d_{i}\right)-H\left(c_{i}\right)\right)+\sum_{i=1}^{n-1} V\left(F ;\left[a_{i}, b_{i}\right] .\right.
\end{aligned}
$$

By (2), (3) and (4), for $n \geq n_{o}$ it follows that

$$
\sum_{i=1}^{n}\left|F\left(d_{i}\right)-F\left(c_{i}\right)\right|>\sum_{i=1}^{n}\left(H\left(d_{i}\right)-H\left(c_{i}\right)\right)-\frac{\alpha}{2} .
$$

Clearly

$$
\sum_{i=1}^{n}\left(H\left(d_{i}\right)-H\left(c_{i}\right)\right)>m(H(Z))=\alpha
$$

hence, by (5)

$$
\sum_{i=1}^{n}\left|F\left(d_{i}\right)-F\left(c_{i}\right)\right|>\frac{\alpha}{2}
$$

By Lemma $4\left(\right.$ since $\left.m^{*}(F(Z))=0\right)$, for each $i=1,2, \ldots, n$ we have

$$
\left|F\left(d_{i}\right)-F\left(c_{i}\right)\right| \leq \sum_{\left\{j:\left[a_{j}, b_{j}\right] \subset\left[c_{i}, d_{i}\right]\right\}} \mathcal{O}\left(F ;\left[a_{j}, b_{j}\right]\right),
$$

hence

$$
\sum_{i=1}^{n}\left|F\left(d_{i}\right)-F\left(c_{i}\right)\right| \leq \sum_{j=n}^{\infty} \mathcal{O}\left(F ;\left[a_{j}, b_{j}\right]\right) .
$$

By (6) and (7) it follows that for each $n \geq n_{o}$ we have

$$
\frac{\alpha}{2} \leq \sum_{j=n}^{\infty} \mathcal{O}\left(F ;\left[a_{j}, b_{j}\right]\right) .
$$

But $\sum_{i=1}^{\infty} \mathcal{O}\left(F ;\left[a_{j}, b_{j}\right]\right) \leq V$, so

$$
\lim _{n \rightarrow \infty} \sum_{j=n}^{\infty} \mathcal{O}\left(F ;\left[a_{j}, b_{j}\right]\right)=0 .
$$

By (8) and (9) we obtain that $\frac{\alpha}{2} \leq 0$, a contradiction. Therefore $H \in(N)$ on $[a, b]$. 
" $\Leftarrow$ " Let $Z \subset[a, b], m(Z)=0$. We may suppose without loss of generality that $Z$ is a $G_{\delta^{-}}$set of the form $Z=\cap_{i=1}^{\infty} G_{i}, G_{i}$ open sets and $G_{1} \supset G_{2} \supset \cdots$. By Lemma 2,

$$
m(H(Z))=m\left(\cap_{i=1}^{\infty} H\left(G_{i}\right)\right)=0 .
$$

Since the sequence of sets $\left\{H\left(G_{i}\right)\right\}_{i}$ is decreasing it follows that

$$
\lim _{i \rightarrow \infty} m\left(H\left(G_{i}\right)\right)=0 .
$$

Let $G_{i}:=\cup_{j=1}^{\infty}\left(a_{j}^{i}, b_{j}^{i}\right)$. For each $i$ we have

$$
\begin{gathered}
m(F(Z))=m\left(F\left(\cup_{j=1}^{\infty}\left(Z \cap\left(a_{j}^{i}, b_{j}^{i}\right)\right)\right) \leq \sum_{j=1}^{\infty} m^{*}\left(F ; Z \cap\left(a_{j}^{i}, b_{j}^{i}\right)\right)<\right. \\
<\sum_{j=1}^{\infty} \mathcal{O}\left(F ;\left[a_{j}^{i}, b_{j}^{i}\right]\right)=\sum_{j=1}^{\infty}\left(H\left(b_{j}^{i}\right)-H\left(a_{j}^{i}\right)\right)=m\left(H\left(G_{i}\right)\right) .
\end{gathered}
$$

Therefore $m(F(Z))=0$.

Lemma 7. Let $F:[a, b] \mapsto \mathbb{R}$ be a continuous and increasing function, satisfying Lusin's condition $(N)$. Then $F \in A C$ on $[a, b]$.

Proof. We shall follow the first part of the proof of the Banach-Zarecki Theorem ([4], p. 250). Suppose that $F \notin A C$ on $[a, b]$. Then there is a number $\epsilon_{o}>0$ having the following property: for every $\delta>0$ there exists a finite family of pairwise disjoint open intervals $\left\{\left(a_{k}, b_{k}\right)\right\}_{k}, k=1,2 \ldots, n$ such that

$$
\sum_{k=1}^{n}\left(b_{k}-a_{k}\right)<\delta \text { and } \sum_{k=1}^{n}\left(F\left(b_{k}\right)-F\left(a_{k}\right)\right) \geq \epsilon_{o} .
$$

Let $\sum_{i=1}^{\infty} \delta_{i}$ be a convergent series of positive terms, and for every $\delta_{i}$, let $\left\{\left(a_{k}^{i}, b_{k}^{i}\right)\right\}_{k}, k=1,2, \ldots, n_{i}$ be a collection of pairwise disjoint open intervals such that

$$
\sum_{k=1}^{n_{i}}\left(b_{k}^{i}-a_{k}^{i}\right)<\delta_{i} \text { and } \sum_{k=1}^{n_{i}}\left(F\left(b_{k}^{i}\right)-F\left(a_{k}^{i}\right)\right) \geq \epsilon_{o} .
$$

Let

$$
E_{i}=\sum_{k=1}^{n_{i}}\left(a_{k}^{i}, b_{k}^{i}\right) \quad \text { and } \quad A=\cap_{i=1}^{\infty}\left(\cup_{i=n}^{\infty} E_{i}\right)
$$


It is easy to verify that $m(A)=0$. By hypotheses it follows that $m(F(A))=0$. Let $G_{n}:=\cup_{i=n}^{\infty} E_{i}$. Then

$$
m\left(F\left(G_{n}\right)\right) \geq m\left(F\left(E_{n}\right)\right)=\sum_{k=1}^{n_{i}}\left(F\left(b_{k}^{i}\right)-F\left(a_{k}^{i}\right) \geq \epsilon_{o} .\right.
$$

By Lemma 2 we have

$$
0=F(A)=m\left(\cap_{n=1}^{\infty} F\left(G_{n}\right)\right)=\lim _{n \rightarrow \infty} m\left(F\left(G_{n}\right)\right) \geq \epsilon_{o}
$$

a contradiction.

Lemma 8. Let $F, H:[a, b] \mapsto \mathbb{R}, H(x)=V(F ;[a, x])$. If $H \in A C$ on $[a, b]$ then $F \in A C$ on $[a, b]$.

Proof. This follows by the fact that $|F(\beta)-F(\alpha)| \leq H(\beta)-H(\alpha)$, whenever $[\alpha, \beta] \subseteq[a, b]$.

The Banach-Zarecki Theorem. Let $F:[a, b] \mapsto \mathbb{R}$. If $F$ is a continuous and $V B$ function, satisfying Lusin's condition $(N)$ then $F$ is $A C$ on $[a, b]$.

Proof. Let $H:[a, b] \mapsto \mathbb{R}, H(x):=V(F ;[a, x])$. By Lemma $6, H$ is continuous and increasing, and satisfies condition $(N)$ on $[a, b]$. By Lemma 7 , $H \in A C$ on $[a, b]$. By Lemma 8 it follows that $F \in A C$ on $[a, b]$.

\section{References}

[1] V. Ene, Real functions - current topics, Lect. Notes in Math., vol. 1603, Springer-Verlag, 1995.

[2] J. Foran, A note on Lusin's condition (N), Fund. Math. 90 (1976), 181186.

[3] J. Foran, Fundamentals of real analysis, Marcel Dekker Inc, New York, Basel, Hong Kong, 1991.

[4] I. P. Natanson, Theory of functions of a real variable, 2nd. rev. ed., Ungar, New York, 1961.

[5] S. Saks, Theory of the integral, 2nd. rev. ed., vol. PWN, Monografie Matematyczne, Warsaw, 1937.

[6] D. E. Varberg, On absolutely continuous functions, Amer. Math. Monthly 72 (1965), 831-841. 